\title{
Needle Insertion Study Using Ultrasound-Based 2D Motion Tracking*
}

\author{
Ehsan Dehghan and Septimiu E. Salcudean \\ Department of Electrical and Computer Engineering, \\ University British Columbia, Canada \\ \{ehsand,tims\}@ece.ubc.ca
}

\begin{abstract}
Needle insertion simulators are in demand to train physicians for surgical procedures such as prostate brachytherapy. In order to design a needle insertion simulator, a needle-tissue interaction model is necessary. Such a model can be identified using force and displacement data measured during the insertion of the needle. In this paper, an experiment is conducted in which a needle is inserted into a two-layered PVC phantom, while the needle position and insertion forces are measured and the tissue is imaged using a trans-rectal probe in the sagittal plane. A $2 \mathrm{D}$ block matching algorithm is used to estimate tissue deformation from the envelope of the recorded radio-frequency signals. This algorithm can be used to estimate the rotation and displacement of the prostate during prostate brachytherapy. The block matching method was validated in an independent experiment. With the measured force, needle position and tissue displacements, a finite element simulation was conducted to identify the parameters of a needle-tissue force distribution model and Young's moduli of each part of the tissue phantom.
\end{abstract}

\section{Introduction}

Prostate Brachytherapy is a common treatment for prostate cancer during which radioactive capsules are implanted in the prostate and surrounding tissue using needles. Accurate placement of the capsules leads to a proper dose and increases the quality of the treatment [1]. However, due to prostate deformation and rotation 2] and lack of visual feedback, accurate needle placement is still a challenge. Brachytherapy simulators have the potential to train physicians and provide preoperative planning. Every needle insertion simulator requires a needle-tissue interaction model. Extensive research has been conducted to provide such a model 3415, including studies on the liver 677 and in-vivo studies on the prostate [8]. In some modeling approaches, only the needle force measurements were used to derive the needle-tissue interaction parameters 68, while in others, tissue displacements were measured and employed in addition to force measurements 3 4 4 5 7]. In 3], 2D displacements of a PVC phantom were measured using surface markers tracked by a camera. By combining the force and displacement data, a

^ This work was supported by NSERC and CIHR.

D. Metaxas et al. (Eds.): MICCAI 2008, Part II, LNCS 5242, pp. 660667 2008.

(C) Springer-Verlag Berlin Heidelberg 2008 
needle force model was introduced that combines a peak at the tip of the needle followed by a constant force distribution along the rest of the needle. Crouch et al. measured the 3D displacement of several markers implanted inside a cube of homogeneous, transparent silicon gel phantom using a stereo-camera system 4 . The force and displacement data were used to identify the parameters of a force distribution model with a peak and a dip at the tip of the needle followed by a constant shaft force density. Hing et al. 7] used a dual C-arm fluoroscope to measure the displacement of fiducial beads inside the tissue in 3D. They identified a local effective modulus during puncture and a cutting force for several samples of tissue. The tissue displacement measurement techniques used by the aforementioned research groups rely on the implanted markers, beads and/or transparency of the tissue.

In our previous work [5], we used ultrasound (US) radio-frequency (RF) signals to estimate the tissue displacement in the US field of view. Ultrasound imaging does not need any beads or markers and is a common imaging modality in many medical procedures including prostate brachytherapy. An experiment was carried out in which a brachytherapy needle was inserted into a PVC prostate phantom. The insertion forces were measured via a load cell and the RF signals were collected using a linear probe mounted at the back of the tissue. Correlationbased methods [910] were used to measure the tissue displacement from the (RF) data. Since the major displacement of the tissue occurred parallel to the axial direction of the US images, the axial tissue displacement was used to identify the unknown parameters of the model. The parameters of a three-parameter force distribution model - tip and shaft force densities and the spacing between them - and the Young's moduli of the two parts of the tissue phantom were identified in a single insertion. During prostate brachytherapy, a trans-rectal ultrasound (TRUS) probe is used to image the tissue in transverse or sagittal/parasagittal planes. If the tissue is imaged in sagittal/parasagittal planes, the major tissue displacement (along the needle insertion axis) occurs in the lateral direction of the US image (lateral and axial directions of the US image and the axis of insertion are shown in Fig. 3(a). Therefore, to study needle-tissue interaction during brachytherapy, one should be able to estimate tissue displacement in the lateral direction with high accuracy. The decoupled correlation methods employed in our prior work did not have high accuracy in the lateral direction due to image de-correlation over large displacements.

In this work, an RF envelope block matching technique is used to estimate tissue deformation in 2D. This method outperforms the RF data cross-correlation method for large lateral displacements. An experiment was carried out in which the TRUS probe was used to image a non-homogeneous PVC tissue phantom during insertion of a needle. This experiment mimics the practical case in which a needle inserted during prostate brachytherapy is imaged with the sagittal (axial) array of the TRUS probe. The most significant tissue displacements, which occur along the needle insertion axis in the lateral direction of the sagittal ultrasound array, the needle position and needle insertion forces are used to model the needle-tissue interaction. In particular, the gathered data is used to identify the 


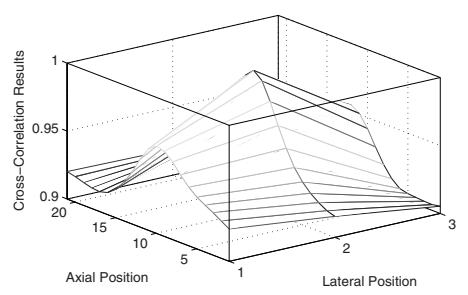

(a)

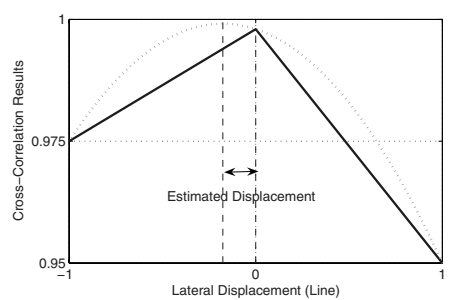

(b)

Fig. 1. (a) a sample cross-correlation matrix and (b) the sub-sampling process

three parameters of the force model introduced in 5 and the Young's moduli of the two-layered tissue phantom. This tissue displacement measurement method can be used in vivo, during prostate brachytherapy, to measure prostate motion and rotation during insertion, which in turn can lead to prostate deformation simulators and path planners for intra-operative treatment planners.

\section{Motion Estimation Algorithm}

The motion estimation algorithm uses the envelope of the RF data. The RF envelope is calculated using:

$$
e_{i}=\sqrt{\left|h\left(R F_{i}\right)\right|}
$$

where $R F_{i}$ is the $i^{\text {th }} \mathrm{RF}$ data line, $h(\cdot)$ denotes the Hilbert transform and $e_{i}$ is the $i^{\text {th }}$ line of the RF envelope. The algorithm tracks the position of the points of interest in several consecutive frames. At first, a Block of Interest (BI) is assumed around every point of interest in the reference frame. Later, the algorithm locates the BIs in the subsequent frames using 2D cross-correlation. For this purpose, each BI is cross-correlated with a Search Region (SR) in the subsequent frames. The position of the BI in each frame and its displacement are found using the maximum values of the cross-correlation matrix. Cross-correlation of the BIs with the entire frame is time consuming and results in multiple false peaks. Therefore, each BI is cross-correlated with a corresponding SR, the size of which is larger than the BI but smaller than the entire frame. The positions of the SRs in the target frame are calculated based on the estimated positions of the corresponding BIs up to the target frame. To find the lateral displacement with higher resolution sub-sampling using quadratic functions was employed [1]. In this method, at first, the maximum value of the cross-correlation matrix is chosen. Later, a quadratic function is fitted to the corresponding row of the matrix. The maximum value of this quadratic function is assumed to correspond to the true displacement. The cross-correlation matrix and sub-sampling processes are shown in Fig, 1 . 


\section{Validation Experiment}

In order to validate the accuracy of the block matching algorithm, a rigid known movement was applied to the tissue phantom and the motion measured using block matching. A linear probe was mounted on a linear stage powered by a DC motor. A PVC phantom was placed inside a box, the front side of which was covered by soft wrapping plastic. The box was filled with water. The probe was placed in touch with the plastic membrane (see Fig.21). In this situation, the probe can move without applying any force on the PVC phantom. The probe was moved sinusoidally $(x(t)=A \sin (\omega t))$ with variable amplitudes and frequencies. The direction of the probe movement was parallel to the lateral direction of the US image. Due to presence of the plastic membrane and water, it can be assumed that the tissue phantom moves rigidly in the lateral direction of the probe. The displacements of several points in the US imaging plane were

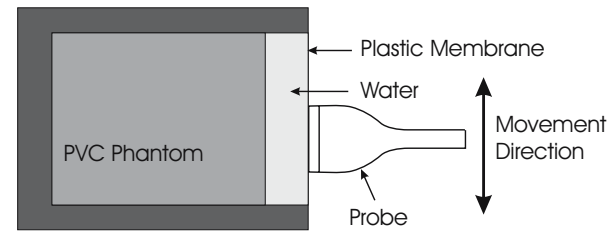

Fig. 2. The relative position of probe and its movement respect to the tissue phantom

measured using the envelope block matching. At each time sample the average of measured displacements was compared with the probe displacement. Therefore, at each time sample, the algorithm showed an error with an average and a standard deviation over the entire US image. Table1 shows the maximum of these values over the time of the experiment (10 seconds for each frequency and amplitude). In our experiments the maximum error occurred at the peak of the displacement and did not increase over time. As expected, the error increases with amplitude and frequency. This table shows the accuracy of the RF envelope block matching in the estimation of large lateral displacements. The linear probe used for the experiment had an array of 128 elements with a density of 2.56 elements per millimeter. The recorded RF frames were up-sampled by a factor of two before the Hilbert transform was applied in order to increase the accuracy of the algorithm. The BIs were three lines in the lateral direction and 56 samples in the axial direction of the image. The SRs were five lines wide and 72 samples long. In the presence of tissue deformation (for example, caused by needle insertion), de-correlation can occur. To compensate for this de-correlation the reference frame was updated every $50^{\text {th }}$ frame.

The motion estimation algorithm under-estimates the lateral displacement if the real movement is less than the line width of the US image. This inaccuracy is due to the fact that the cross-correlation of one BI in the reference frame and the same size blocks in the SR is not only a function of the spatial proximity of 
Table 1. Validation results for lateral motion estimation algorithm

\begin{tabular}{l|c|c|c|c|c}
\hline Frequency $(\mathrm{Hz})$ & 0.25 & 0.5 & 0.25 & 0.25 & 1.0 \\
\hline Peak to peak $(\mathrm{mm})$ & 4.0 & 2.0 & 2.0 & 6.0 & 2.0 \\
\hline Max. Ave. Error $(\mathrm{mm})$ & 0.26 & 0.14 & 0.11 & 0.23 & 0.30 \\
\hline Max. StD. Error $(\mathrm{mm})$ & 0.39 & 0.28 & 0.24 & 1.3 & 0.14 \\
\hline
\end{tabular}

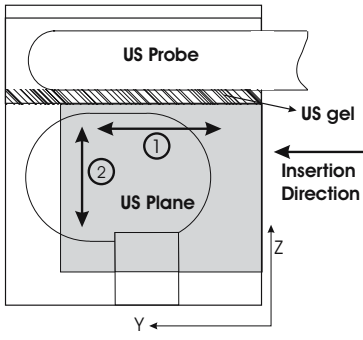

(a)

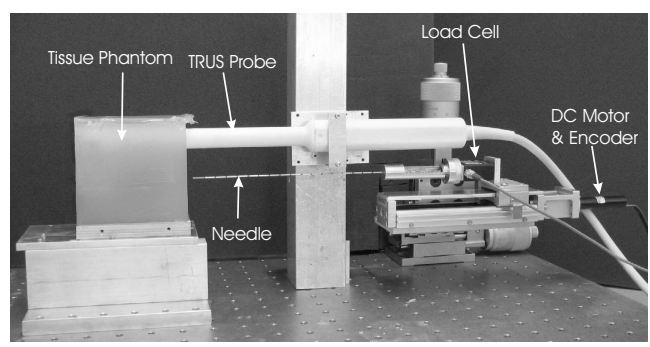

(b)

Fig. 3. (a) The cross-section of the tissue phantom, showing the US field of view. Arrows 1 and 2 show the lateral and axial directions in the US image, respectively. In the shown configuration, the US plane coincides with the sagittal plane of the tissue.

(b) The experimental setup.

the two blocks, but also a function of their texture similarity. This fact also leads to non-zero motion estimation when the tissue is stationary. In this situation, the cross-correlation matrix is asymmetric respect to its middle column due to asymmetric texture of the SR. The asymmetric cross-correlation matrix leads to a non-zero motion displacement after the sub-sampling process is applied.

\section{Needle Insertion Experiment}

An experiment was conducted to record the insertion force and tissue displacement data during insertion of a needle into a two-layered prostate phantom. In this experiment, a brachytherapy needle (Bard, USA) was mounted on a linear stage powered by a DC motor equipped with an optical encoder. The axial insertion forces were measured using a 1 DoF load cell (MBD-2.5 Transducer Techniques, USA) housed at the base of the needle. A two-layered prostate phantom was constructed which was composed of a hard inclusion (50\% plastic hardner and 50\% PVC plasticizer) and a softer substrate (33\% plastic softner and $67 \%$ PVC plasticizer). Cellulose was added to both parts to produce scatterers. A PC-based Sonix RP (Ultrasonix Medical Corp., Canada) ultrasound machine was used to image the tissue in the sagittal plane using a TRUS probe located inside a hollow cylinder in the phantom (see Fig.3(a). The experimental setup is shown in Fig. 3(b)

The needle was inserted with a constant speed of $5 \mathrm{~mm} / \mathrm{s}$. The needle stylet was fully inserted to create a symmetric tip. No significant needle bending was 


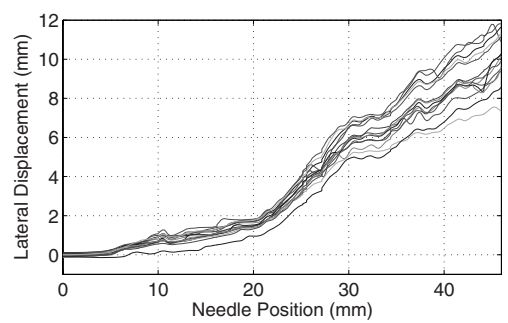

(a)

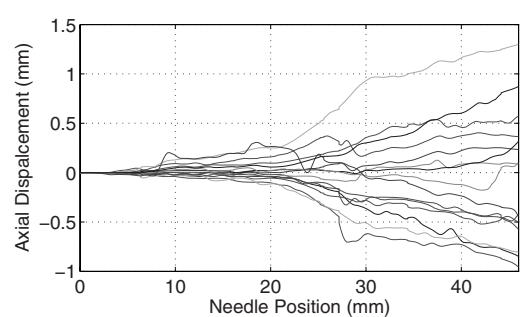

(b)

Fig. 4. Displacements of the mesh nodes (a) along the axis of insertion (lateral direction of the US image) and (b) along the axial direction of the US images

Table 2. Needle shaft force distribution and elastic parameters

\begin{tabular}{lcccc}
\hline & $f_{\mathrm{s}}(\mathrm{N} / \mathrm{m})$ & $f_{\mathrm{p}}(\mathrm{N} / \mathrm{m})$ & $w(\mathrm{~mm})$ & Young's Modulus $(\mathrm{kPa})$ \\
\hline Inclusion & 90 & 380 & 4.5 & 30 \\
Surrounding tissue & 50 & 100 & 4.0 & 7 \\
\hline
\end{tabular}

observed during the insertion. The TRUS probe imaged the tissue to the depth of $50 \mathrm{~mm}$ with 128 elements in the lateral direction $(60 \mathrm{~mm})$ at a rate of 25 frames per second. The needle position, the insertion force and a time stamp were sent to the US machine computer via a serial port. In a loop, the US machine records the $\mathrm{RF}$ data and then reads the values of the load cell, needle position and the accompanying time stamp from the serial port. Since the tissue phantom was meshed to be used in an FEM analysis, the displacements of mesh nodes located on the US plane were measured in 2D using the block matching algorithm. The lateral and axial displacement of the mesh nodes as functions of time are shown in Fig.4. The position of the mesh nodes in the US image are shown in Fig. $5(\mathrm{~b})$

\section{Results}

Similarly to the method presented in [5], the experimental data were used in a linear FEM-based needle insertion simulator (for more detail please see [5]). The three parameters of the force distribution model (peak force, shaft force, and width of the peak force) were adopted to match the simulated and measured insertion forces. The Young's moduli of the two parts of the phantom were identified to match the average of the simulated and measured displacement along the $y$ axis (the major displacement). The PVC phantom Poisson's ratio was taken to be 0.49 to simulate a nearly incompressible material. The identified parameters are presented in Table2. The simulated and measured forces are shown in Fig.5(a). The original positions of the mesh nodes in the US image and the measured and simulated displaced positions are shown in Figs.5(b) and 5(c).

The accuracy of the identified parameters depends on the accuracy of the simulation and the measured data. The accuracy of the simulation can be increased by using finer meshes and/or non-linear models (i.e. neo-Hookean) at the cost 


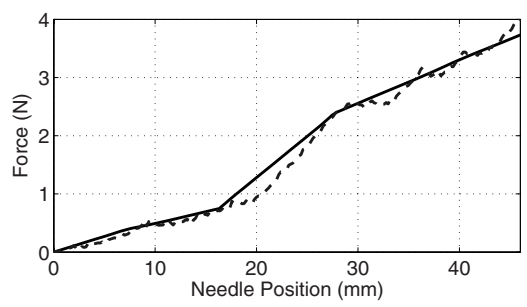

(a)

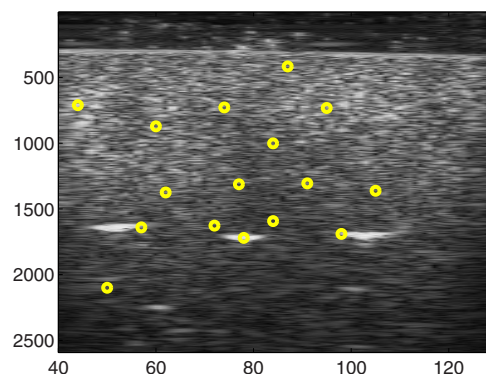

(b)

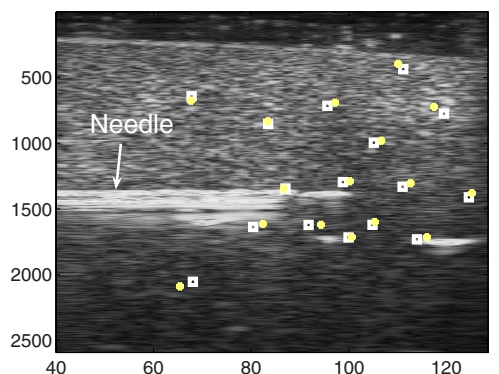

(c)

Fig. 5. (a) Simulated and measured insertion forces vs. needle tip position (needles moves from the left to the right), (b) The original positions of the mesh nodes and (c) the measured (squares) and simulated (circles) positions of the displaced nodes

of higher computational time. The identified Young's moduli may be different from the real Young's moduli of the tissue. However, for simulation purposes, they are acceptable as long as they yield accurate simulation results.

The 2D tracking algorithm showed high accuracy in tracking the large lateral displacements. However, lateral displacements of less than one line width were under-estimated. Without sub-sampling in the lateral direction, the algorithm shows sharp jumps when the displacement is larger than one line width. Sub-sampling creates a continuous output. Due to under-estimation of small displacements, the reference frame update rate should be chosen carefully. Very high update rates lead to large errors, since at each time sample the displacement of the current frame with respect to the reference frame can be less than one line. Very low update rates lead to de-correlation and poor tracking. The optimal reference update rate requires further study. A PVC phantom was used to make the experiment repeatable. However, insertion forces in this phantom do not show the sharp dips observable during insertion of the needle into real tissue. This may be due to the absence of a hard capsule in the phantoms. The extension of this work to real tissue will be undertaken in the future.

\section{Conclusion and Future Work}

In this paper a $2 \mathrm{D}$ block matching algorithm was used to measure the displacement of a tissue phantom during needle insertion. The algorithm estimates the 
displacement using 2D correlation maximization of the RF envelopes of the reference and target frames. The accuracy of the algorithm in measuring the lateral motion was tested during an experiment in which a linear probe was used to measure the rigid lateral motion of a PVC phantom. During a needle insertion experiment a two-layered PVC phantom was imaged using a TRUS probe in the sagittal plane. The tissue displacement data, the needle position and the insertion force measured during the experiment were fed to an FEM needle insertion simulator to identify the parameters of a needle-tissue interaction model [5].

In the future, this method will be used during prostate brachytherapy to measure the prostate rotation and deformation during needle insertion. The in-vivo measurements can be used later to generate a needle-tissue interaction model to be used in brachytherapy simulators and path planners.

\section{References}

1. Dawson, J.E., Wu, T., Roy, T., Gu, J.Y., Kim, H.: Dose effects of seeds placement deviation from pre-planned position in ultrasound guided prostate implants. Radiotherapy and Oncology 32(2) (1994)

2. Lagerburg, V., Moerland, M.A., Lagendijk, J.J., Battermann, J.J.: Measurement of prostate rotation during insertion of needles for brachytherapy. Radiotherapy and Oncology 77, 318-323 (2005)

3. DiMaio, S.P., Salcudean, S.E.: Needle insertion modeling and simulation. IEEE Trans. on Robot. Autom.: Special Issue on Medical Robotics 19, 864-875 (2003)

4. Crouch, J.R., Schneider, C.M., Wainer, J., Okamura, A.M.: A velocity-dependent model for needle insertion in soft tissue. In: Duncan, J.S., Gerig, G. (eds.) MICCAI 2005. LNCS, vol. 3750, pp. 624-632. Springer, Heidelberg (2005)

5. Dehghan, E., Wen, X., Zahiri-Azar, R., Marchal, M., Salcudean, S.E.: Modeling of needle-tissue interaction using ultrasound-based motion estimation. In: Ayache, N., Ourselin, S., Maeder, A. (eds.) MICCAI 2007, Part I. LNCS, vol. 4791. Springer, Heidelberg (2007)

6. Okamura, A., Simone, C., O'Leary, M.: Force modeling for needle insertion into soft tissue. IEEE Trans. Biomed. Eng. 51, 1707-1716 (2004)

7. Hing, J.T., Brooks, A.D., Desai, J.P.: A biplanar fluoroscopic approach for the measurement, modeling, and simulation of needle and soft-tissue interaction. Medical Image Analysis 11, 62-78 (2007)

8. Podder, T., Sherman, J., Messing, E., Rubens, D., Fuller, D., Strang, J., Brasacchio, R., Yu, Y.: Needle insertion force estimation model using procedure-specific and patient-specific criteria. In: Proc. IEEE EMBS Int. Conf., pp. 555-558 (2006)

9. Zahiri-Azar, R., Salcudean, S.E.: Motion estimation in ultrasound images using time domain cross correlation with prior estimates. IEEE Trans. Biomed. Eng. 53(10), 1990-2000 (2006)

10. Zahiri-Azar, R., Salcudean, S.E.: Real-time estimation of lateral motion using time domain cross correlation with prior estimates. In: Proc. IEEE Ultrasonics Symposium, pp. 1209-1212 (2006)

11. Cespedes, I., Huang, Y., Ophir, J., Spratt, S.: Methods for the estimation of subsample time-delays of digitized echo signals. Ultrasonic Imaging 17, 142-171 (1995) 